\title{
Article \\ On the Equivalence of the Biological Effect Induced by Irradiation of Clusters of Heavy Atom Nanoparticles and Homogeneous Heavy Atom-Water Mixtures
}

\author{
Balder Villagomez-Bernabe ${ }^{1, * \mathbb{C}}$, José Ramos-Méndez ${ }^{2} \mathbb{D}$ and Frederick J. Currell ${ }^{1}$ \\ 1 The Dalton Cumbria Facility and the School of Chemistry, The University of Manchester, Oxford Rd, \\ Manchester M13 9PL, UK; frederick.currell@manchester.ac.uk \\ 2 Department of Radiation Oncology, University of California San Francisco, 1600 Divisadero Street, \\ San Francisco, CA 94115, USA; jose.ramosmendez@ucsf.edu \\ * Correspondence: balder.villagomez-bernabe@manchester.ac.uk
}

check for updates

Citation: Villagomez-Bernabe, B.; Ramos-Méndez, J.; Currell, F.J. On the Equivalence of the Biological Effect Induced by Irradiation of Clusters of Heavy Atom Nanoparticles and Homogeneous Heavy Atom-Water Mixtures. Cancers 2021, 13, 2034. https://doi.org/10.3390/cancers 13092034

Academic Editor: David Wong

Received: 30 March 2021

Accepted: 21 April 2021

Published: 23 April 2021

Publisher's Note: MDPI stays neutral with regard to jurisdictional claims in published maps and institutional affiliations.

Copyright: (C) 2021 by the authors Licensee MDPI, Basel, Switzerland. This article is an open access article distributed under the terms and conditions of the Creative Commons Attribution (CC BY) license (https:// creativecommons.org/licenses/by/ $4.0 /)$.
Simple Summary: The use of nanoparticles in radiotherapy has been studied widely for over a decade due to their ability to reduce the survival fraction of tumor cells while reducing the doses deposited in healthy cells. Mathematical models were successfully implemented to reproduce experimental results in the literature at the $\mathrm{keV}$ range, but discrepancies were found at the MV energy range, the latter range being most used in radiotherapy. The main finding of this work is the demonstration of an equivalence of the physically mediated component of the cell damage between a cluster of nanoparticles and a gold-water mixture in the MV energy range, which reduces the complexity of modeling the interactions of radiation with clusters of nanoparticles seen in real case scenarios.

Abstract: A multiscale local effect model (LEM)-based framework was implemented to study the cell damage caused by the irradiation of clusters of gold nanoparticles (GNPs) under clinically relevant conditions. The results were compared with those obtained by a homogeneous mixture of water and gold (MixNP) irradiated under similar conditions. To that end, Monte Carlo simulations were performed for the irradiation of GNP clusters of different sizes and MixNPs with a 6 MV Linac spectrum to calculate the dose enhancement factor in water. The capabilities of our framework for the prediction of cell damage trends are examined and discussed. We found that the difference of the main parameter driving the cell damage between a cluster of GNPs and the MixNP was less than $1.6 \%$ for all cluster sizes. Our results demonstrate for the first time a simple route to intuit the radiobiological effects of clusters of nanoparticles through the consideration of an equivalent homogenous gold/water mixture. Furthermore, the negligible difference on cell damage between a cluster of GNPs and MixNP simplifies the modelling for the complex geometries of nanoparticle aggregations and saves computational resources.

Keywords: nanoparticle; cytoplasm; radiotherapy; nanomedicine

\section{Introduction}

Nanoparticles of high atomic number have been widely used in medicine as contrast agents and nano-carriers due to their biocompatibility and small size [1,2]. Furthermore, since Hainfeld's studies in 2004 on the use of nanoparticles as radiosensitizers [3], considerable effort has been made to understand the mechanisms of physical interactions between ionizing radiation and nanoparticles. The role of such nanoparticles in biomedical imaging, cancer therapy, and biophysics has been reviewed elsewhere [4,5]. Theoretical models [6,7] have predicted that the maximum physical enhancement should be seen at keV energies, where the highest values for the ratio of the mass energy absorption coefficient between 
gold and water were calculated. At that energy range, the photoelectric effect is the predominant physical process, which leads to the generation of a cascade of low energy Auger electrons during the deexcitation process after each interaction; those Auger electrons are mainly responsible for depositing a high quantity of energy around the nanoparticle, seen as spikes of localized dose in the cell near or hosting the nanoparticle.

Nevertheless, as Butterworth et al. [8] have summarized, previous theoretical models failed to predict the radiosensitization enhancement observed in experiments in vitro for MV irradiation. Those models assumed (implicitly) a uniform distribution of the nanoparticles inside the cells, neglecting mutual interactions between them. However, it has been shown that nanoparticles tend to aggregate inside the tumor [9-11], leading to heterogeneous distributions even inside the cells, suggesting the necessity for further studies on the change in radiobiological response due to clustering of nanoparticles. We therefore applied the local effect model to perform a multiscale approach under relevant clinical conditions in order to investigate the radiosensitization enhancement in cells due to clusters of gold nanoparticles (GNPs) of different sizes and positions relative to the radiation-sensitive structures inside the cell.

The effects of nanoparticles on DNA are currently a matter for discussion in the scientific literature, see e.g., [12,13]. Furthermore, it is not clear that the only critical subcellular target is nuclear DNA. Possible critical targets located in the cytoplasm include mitochondria and lysosomes [14,15].

Due to the complexity of GNP distribution inside the tumor cell, previous works in the literature $[16,17]$ have approximated the clusters with a homogeneous mixture of water and gold. A review of recent progress in gold nanoparticle dosimetry can be found in [18] To the best of our knowledge, no benchmarking against the presence of clusters of GNPs has yet been carried out to compare the effects at the biological stage. Thus, in this work we carried out a set of Monte Carlo simulations to test the hypothesis of the equivalence of the biological effect generated by dose enhancement at the physical stage between clusters of nanoparticles and homogenous mixtures. The concepts underlying this comparison are shown in Figure 1.

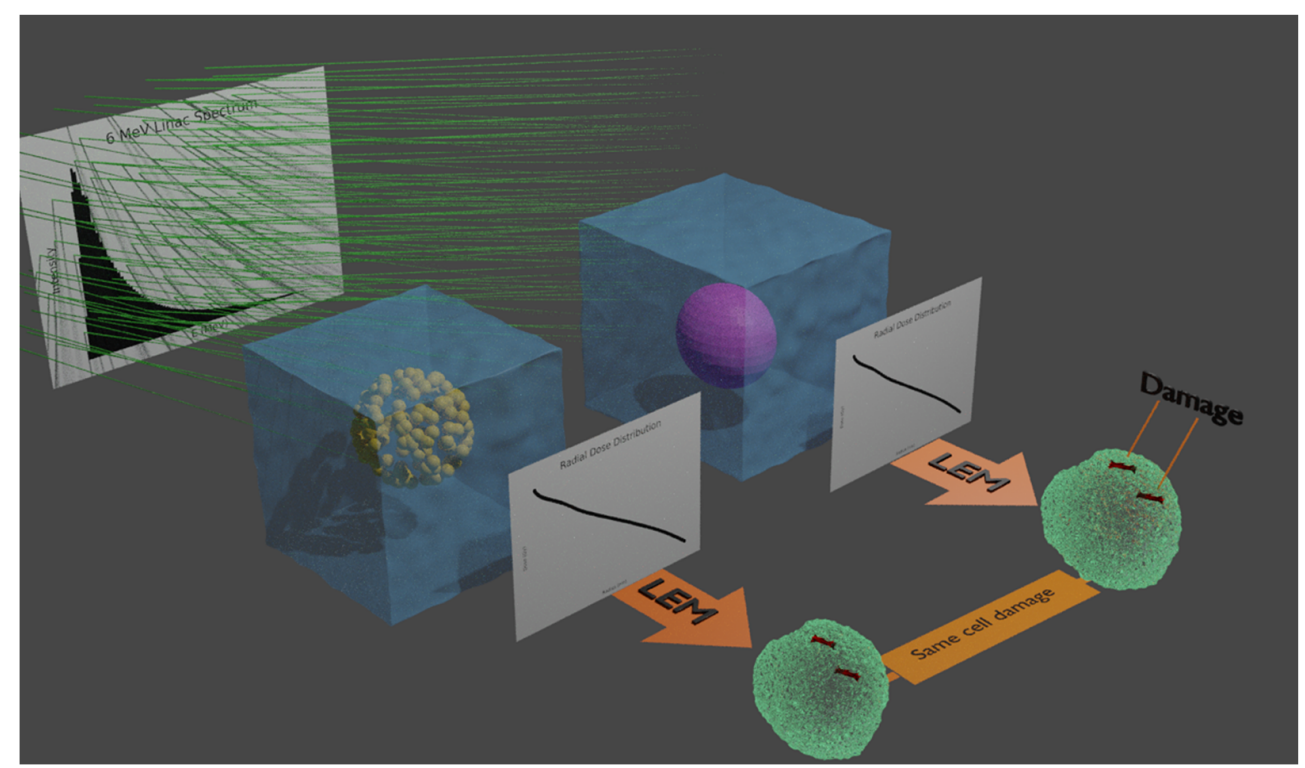

Figure 1. The histogram at the back shows the $6 \mathrm{MV}$ Linac spectrum used to irradiate both a cluster of gold nanoparticles (GNPs) and the MixNP (homogeneous mix of gold and water) in order to calculate radial dose distributions (RDDs) in water (black curves). The cluster of GNPs is represented by many small gold spheres in the left-most water cube, whilst the gold-water mixture comprising the MixNP is represented by the magenta sphere in the right-most water cube. The RDDs were inserted into the local effect model (LEM) framework, represented as orange arrows, for the calculation of the biological effect. 


\section{Materials and Methods}

The multiscale approach performed in this work involved the prediction of the biological effect from the interactions between ionizing radiation and GNPs/MixNP. Although the calculations presented are concerned with GNPs, the modelling of physical processes will be similar for any nanoparticle containing a significant fraction of heavy atoms. Hence, the results are applicable to this wider class of nanoparticles.

\subsection{Physical Stage}

We performed a set of Monte Carlo simulations consisting of condensed-history Monte Carlo with Geant4, suitable for macroscale; and track-structure Monte Carlo with Geant4-DNA, suitable for micro and nanoscale. The different physics lists and relevant parameters for the electromagnetic processes used in each simulation are shown in Table 1. Deexcitation processes including Auger, Auger cascade, and fluorescence, were activated for all simulations.

Table 1. Parameter values for each simulation performed at the physical stage.

\begin{tabular}{cccc}
\hline Parameters & \multicolumn{2}{c}{ MC Condensed History } & MC Track-Structure \\
\hline Physics list & Livermore & Livermore & Geant4-DNA \\
Energy cut $(\mathrm{eV})$ & 100 & 10 & 10 \\
Range cut $(\mathrm{nm})$ & 1000 & 1 & 1 \\
Max step $(\mathrm{nm})$ & 1000 & 1 & 1 \\
Used for calculating & PHSP1 & PHSP2 & RDD \\
\hline
\end{tabular}

The simulation at the macroscale involved the transport of $\mathrm{X}$-rays through a water phantom of $20 \times 20 \times 40 \mathrm{~cm}^{3}$. To that end, the initial energy spectrum was taken from a 6 MV TrueBeam Linac set in Flattening-Filter mode to produce a $10 \times 10 \mathrm{~cm}^{2}$ field at $90 \mathrm{~cm}$ source-surface distance (SSD), obtained elsewhere [19]. The X-rays traveled in the water phantom and a phase space file (PHSP1) was generated in a circular plane of $0.25 \mathrm{~cm}$ radius, placed at the depth of the maximum dose. The phase space file stored the information of all particles, charged and neutral, crossing that plane. For the simulation at the nanoscale, the spatial coordinates of the phase space were scaled down to the nanoscale as explained in [20]. Later, the PHSP1 was used as a particle source to irradiate the cluster of nanoparticles and the mixture of water and gold. In order to reduce statistical uncertainty for the radial dose distribution (RDDs) below $2 \%$ for all radial bins, the phase space file was recycled 30, 50, and 200 times for the 500, 250, and $70 \mathrm{~nm}$ clusters, respectively.

The three cluster sizes considered in this work consisted of 333 GNPs of 7, 25, and $50 \mathrm{~nm}$ radius. The GNPs were distributed in a randomly uniform distribution without overlapping, ensuring that all of them were all bounded inside a big water nanoparticle (WNP) of 70, 250, and $500 \mathrm{~nm}$ radius, respectively. After irradiating the cluster using PHSP1, a new phase space (PHP2) was recorded at the outer surface of the WNP with the information of only those electrons that were created inside the cluster and able to escape from it. The simulations for the full cluster were repeated ten times using different random seeds.

Three MixNPs were modelled as spheres of radius 70, 250, and $500 \mathrm{~nm}$ made of a homogeneous mixture of gold and water with density of $7.1 \mathrm{~g} / \mathrm{cm}^{3}$. The density was calculated, taking into account the mass of water and gold inside the cluster over the entire volume. We performed the simulations using the PHSP1 as a source to irradiate the MixNPs, generating a new PHSP2, similarly to the process used for the full cluster. Ten simulations were run using different random seeds.

Lastly, two independent track-structure simulations were performed using the Geant4DNA physics list [21-23] to calculate the dose enhancement in water by GNPs and MixNP activation using their corresponding PHSP2s, as calculated previously. Each PHSP2 was positioned at the center of a water sphere of $1 \mathrm{~mm}$ radius. Multiple concentric spherical scorers were placed to record RDD in the sphere. To speed up the simulations, the thickness 
of the spherical scorers was set as shown in Table 2. Ten simulation jobs were launched for both the MixNP and the cluster of GNPs in order to obtain the mean value of the RDDs with their associated standard error.

Table 2. Bin sizes used for the calculation of RDDs in water for each cluster size.

\begin{tabular}{|c|c|c|c|}
\hline \multirow{2}{*}{ Bin Size [nm] } & \multicolumn{3}{|c|}{ Range [nm] for Each Cluster Size } \\
\hline & $70 \mathrm{~nm}$ & $250 \mathrm{~nm}$ & $500 \mathrm{~nm}$ \\
\hline 1 & $70-300$ & $250-500$ & 500-1000 \\
\hline 10 & $300-1 \times 10^{3}$ & $500-1 \times 10^{3}$ & - \\
\hline 100 & $1 \times 10^{3}-1 \times 10^{4}$ & $1 \times 10^{3}-1 \times 10^{4}$ & $1 \times 10^{3}-1 \times 10^{4}$ \\
\hline $1 \times 10^{3}$ & $1 \times 10^{4}-1 \times 10^{5}$ & $1 \times 10^{4}-1 \times 10^{5}$ & $1 \times 10^{4}-1 \times 10^{5}$ \\
\hline $1 \times 10^{4}$ & $1 \times 10^{5}-1 \times 10^{6}$ & $1 \times 10^{5}-1 \times 10^{6}$ & $1 \times 10^{5}-1 \times 10^{6}$ \\
\hline
\end{tabular}

\subsection{Biological Stage}

We modelled a biological cell as two concentric spheres of 15 and $7 \mu \mathrm{m}$ radius, representing the cell and nucleus, respectively. The placement of the cluster varied from 7 to $40 \mu \mathrm{m}$ from the nucleus surface to study cell damage as a function of the relative distance between the cluster/MixNP and the volumes of interest. The lower limit was chosen to avoid the placing of the cluster inside the nucleus, while the upper limit was set to study how cluster distance influences damage to nearby cells.

\subsection{Software and Hardware Specifications}

The software used to perform the Monte Carlo simulations of the transport of ionizing radiation through matter was TOPAS (Tool for Particle Transport Simulation) [24] version 3.2, which is built on top of the Geant4 toolkit [25] version 10.5. TOPAS wraps and extends Geant 4 with a simplified syntax.

The Monte Carlo simulations were carried out using the HPC Pool facilities at the University of Manchester with the following specifications: $128 \times 32$ compute nodes, each node involving $2 \times 16$-core Intel Skylake Gold 6130 CPUs at $2.10 \mathrm{GHz}$ and $192 \mathrm{~GB}$ RAM.

\section{Results}

We studied the difference in cells damage derived from dose enhancement by a cluster of GNPs and a gold/water mixed nanoparticle (MixNP) activated with X-ray irradiation. To compare the differences in the physical and biological stages between them, the percentage of the difference (\%Diff) of all the parameters of interest were calculated using Equation (1).

$$
\% \text { Diff }=\left(X_{\text {mixNP }}-X_{\text {cluster }}\right) * 100 / X_{\text {cluster }},
$$

where $X_{\text {mixNP }}$ and $X_{\text {cluster }}$ represent any pertinent observable associated with the MixNP and a cluster of GNPs, respectively. Thus, a negative percentage difference suggests a higher value of the observable for the cluster than that for the MixNP.

\subsection{Physical Stage: The Monte Carlo Simulations}

The results of the physical stage obtained through Monte Carlo simulations involving the interaction of a cluster of GNPs with X-ray irradiation are shown in Figure 2. The left column shows the energy spectra of the secondary electrons that were created inside the cluster and were able to exit from it, for the three different cluster sizes (70, 250, and $500 \mathrm{~nm})$. The right column illustrates a comparison to the dose enhancement factors (DEFs). For any set of conditions, the DEF is defined in Equation (2) as the ratio of the dose deposited in the water phantom measured in a given radial bin over the dose in the same radial bin when the cluster/MixNP is replaced with a water nanoparticle of the same size, i.e.,

$$
\operatorname{DEF}(r)=\operatorname{Dose}_{G N P}(r) / \operatorname{Dose}_{W N P}(r),
$$


where, $\operatorname{Dose}_{G N P}(r)$ and $\operatorname{Dose}_{W N P}(r)$ are the dose deposited in the radial bin $r$ by a cluster/MixNP and a WNP, respectively.
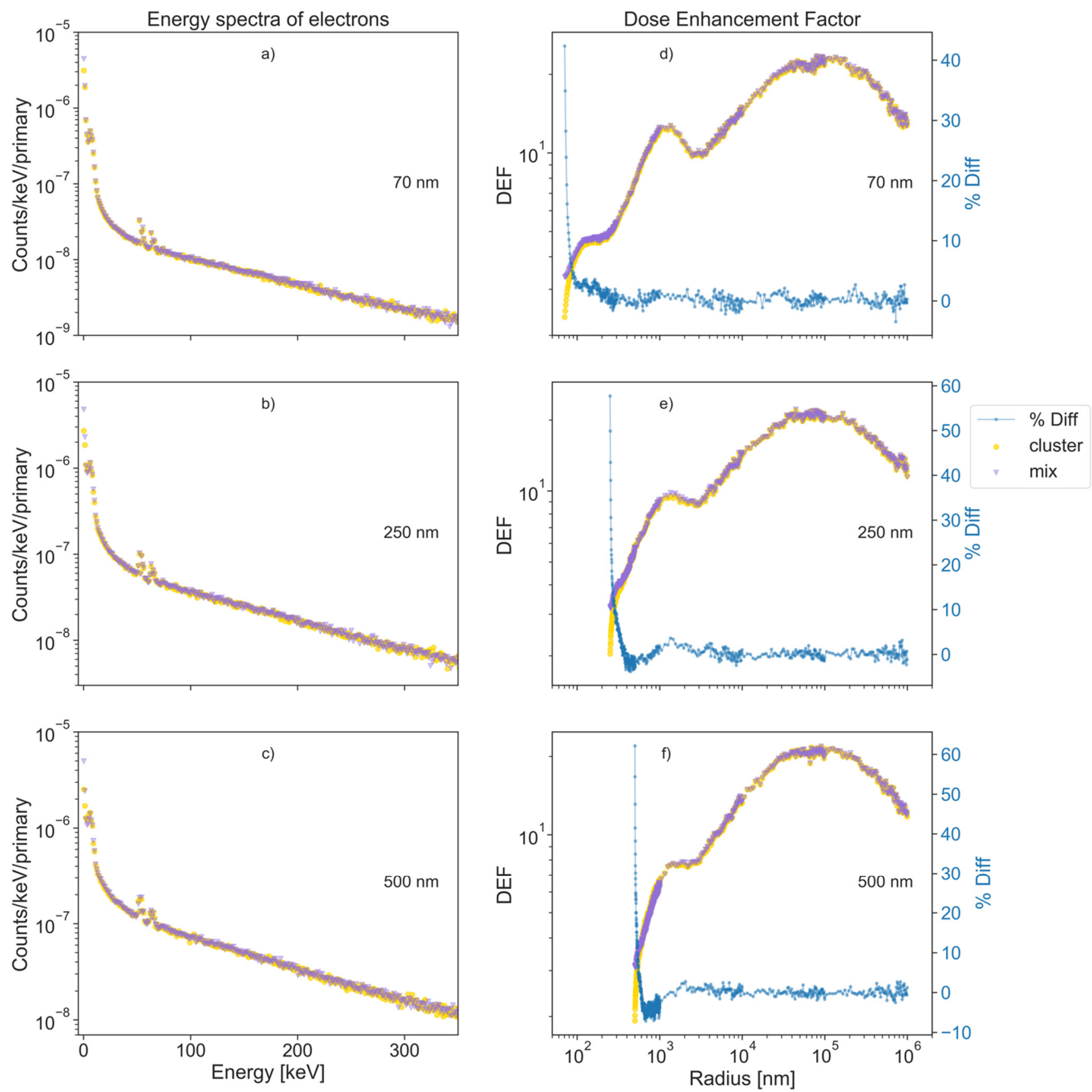

Figure 2. Three different cluster sizes of 70, 250, and $500 \mathrm{~nm},(\mathbf{a}-\mathbf{f})$ each made of 333 GNPs of 7, 25, and 50 nm radii, respectively (top to bottom), were irradiated with a $6 \mathrm{MV}$ energy spectrum from a TrueBeam Varian Linac. Left columns (a-c) show the energy spectra of electrons created inside the cluster and a MixNP that escaped from it (yellow and purple, respectively), for all cluster sizes. Here, the highest difference between both spectra is at the first energy bin, which corresponds to energies below $0.1 \mathrm{keV}$. In the right column, (d-f) show the DEF of the MixNP and the full cluster, and also the \%Diff (in blue) for a better visualization of the differences on the DEF for all sizes. Here, we observed that a higher number of low-energy electrons from the MixNP leads to an enhancement of the DEF at small radii, compared to the DEF of the cluster. The high values of the \%Diff at small radii are associated to the models used to simulate full clusters, where the gold concentration decreases at the surface of the cluster. It is shown in the Supplementary Material that the values of the $\%$ Diff were reduced dramatically when a more complex MixNP was modelled as two concentric spheres made of water and a mix of gold plus water for the outer and the inner sphere, respectively. 
The left column in Figure 2 shows that the energy spectrum of the secondary electrons leaving the cluster was very similar to that escaping from the MixNP, except at lower energies. The maximum difference was found in the first bin, centered at $0.1 \mathrm{keV}$, where MixNP produced 42, 77, and 97\% more electrons than a full cluster for the radii of 70, 250, and $500 \mathrm{~nm}$ respectively. For better visualization, the energy spectrum was plotted up to $350 \mathrm{keV}$, as only a low percentage of electrons have energies greater than that value $(3.7,6.7$ and $8.6 \%$ for the cluster sizes of 70,250 , and $500 \mathrm{~nm}$ radii, respectively).

The right column in Figure 2 illustrates the influence the percentage difference of the energy spectrum of electrons at low energy, as mentioned above, has on the dose enhancement factor (DEF), in water. In the figure, it is observed that the higher values of the $\%$ Diff for the DEF occurred at shorter radial distances. The maximum difference occurred at the first radial bin, where it took values of 42,58 , and $62 \%$, and decreased sharply down to $5 \%$ at 15,55 , and $50 \mathrm{~nm}$ for the 70,250 , and $500 \mathrm{~nm}$ cluster sizes, respectively. For better visualization of such difference, we plotted the \%Diff obtained from Equation (1). The high values of the \%Diff at small radius can be associated with the geometrical model used to simulate the full cluster, where all the gold nanoparticle positions were fixed to be entirely inside the boundary of the cluster. This condition led to a reduction in the average amount of gold material near the surface of the cluster, in contrast with the constant gold density of the MixNP. This effect is illustrated in Figures S1-S3, in the Supplementary Materials, where MixNP was modelled as two concentric spheres made of water and a mix of gold plus water for the outer and inner spheres, respectively, using the parameters in Table S1, leading to a considerable decline of the \%Diff near the cluster surface. However, when the biological effects are considered, there is no benefit to using this more complicated structure for the MixNP, so a simple homogenous spherical geometry was used for the majority of this study. The "waviness" of the DEF was directly correlated to the energy of the secondary electrons produced by the photoelectric effect inside the nanoparticle [26], which not only generated photoelectrons but also initiated an Auger cascade of low-energy electrons during the atomic deexcitation.

\subsection{Biological Stage: The Local Effect Model}

We applied the local effect model described in [27] to predict the average number of lethal lesions $\left(N_{t o t}\right)$ in the cell, as shown below:

$$
\left\langle N_{\text {total }}\right\rangle=\alpha+\beta D^{2}+\left(\alpha D I_{1}+\beta D I_{2}+2 \beta D^{2} I_{1}\right) / V_{\text {sens }},
$$

where $\alpha$ and $\beta$ are experimental parameters that depend on the cell line, while $D$ represents the dose deposited in point $r$ (in the absence of GNPs), and $V_{\text {sens }}$ is the sensitive volume over which the lethal lesions are calculated. Here, $I_{1}$ and $I_{2}$ are the main parameters for the prediction of the $N_{\text {total }}$ due to nanoparticle activation with $X$-rays. By considering the cell as two concentric spheres (cytoplasm and nucleus), where a spherical cluster of nanoparticles is placed inside the cytoplasm, we can exploit the symmetry of this system and approximate $I_{1}$ and $I_{2}$, as in [28]:

$$
\begin{aligned}
& I_{1}=\int D(r) d V \approx \int \frac{\Omega(r)}{4 \pi} S_{1}(r) d r, \\
& I_{2}=\int D^{2}(r) d V \approx \int \frac{\Omega(r)}{4 \pi} S_{2}(r) d r,
\end{aligned}
$$

where the function $\Omega(r)$ represents the solid angle between the center of the cluster/MixNP and the volume of interest at the radial distance $r$. Here, we neglected the dependency on the angles because the cluster has no specific orientation within the volume of interest or radiation field. Here $S_{1}$ and $S_{2}$ are defined as:

$$
S_{1}(r)=\int_{S} D(r) d A
$$




$$
S_{2}(r)=\int_{S} D^{2}(r) d A
$$

Equations (6) and (7) were solved numerically to calculate $S_{1}$ and $S_{2}$, where the RDDs scored from the Monte Carlo simulations were used as input parameters. $\Omega(r)$ was calculated analytically for the two regions of consideration (nucleus and cytoplasm) by exploiting the spherical symmetry of the cell, as explained in [28]. Parameters $I_{1}$ and $I_{2}$ were calculated for both regions of interest by substituting $S_{1}, S_{2}$, and $\Omega(r)$ in Equations (4) and (5) and solving them numerically. We repeated the same procedure for the MixNP in order to obtain the percentage difference (\%Diff) between them, using Equation (1) to investigate the difference in cell damage caused by the cluster of GNPs with respect to the MixNP.

Figure 3 shows the behavior of the parameters $S_{1}$ and $S_{2}$ defined in Equations (6) and (7). A steady decrease of $S_{1}$ (associated with the $r^{2}$ dependence of the area), led to a necessity to score the dose deposited in the cell at far distances from the cluster. The highest values of the $\%$ Diff for $S_{1}$ in the first radial bin were 42,57 , and $62 \%$ (smallest to largest clusters). However, this difference quickly fell to $5 \%$ for the radial distances of 15 , 60 , and $50 \mathrm{~nm}$ beyond the cluster surfaces for the 70,250, and $500 \mathrm{~nm}$ sizes, respectively. The highest values of the $\%$ Diff for $S_{2}$ were 102,149, and $163 \%$ in the first radial bin. These differences fell to $5 \%$ at 26,80 , and $73 \mathrm{~nm}$ beyond the cluster surfaces for the of 70,250 , and $500 \mathrm{~nm}$ sizes, respectively.
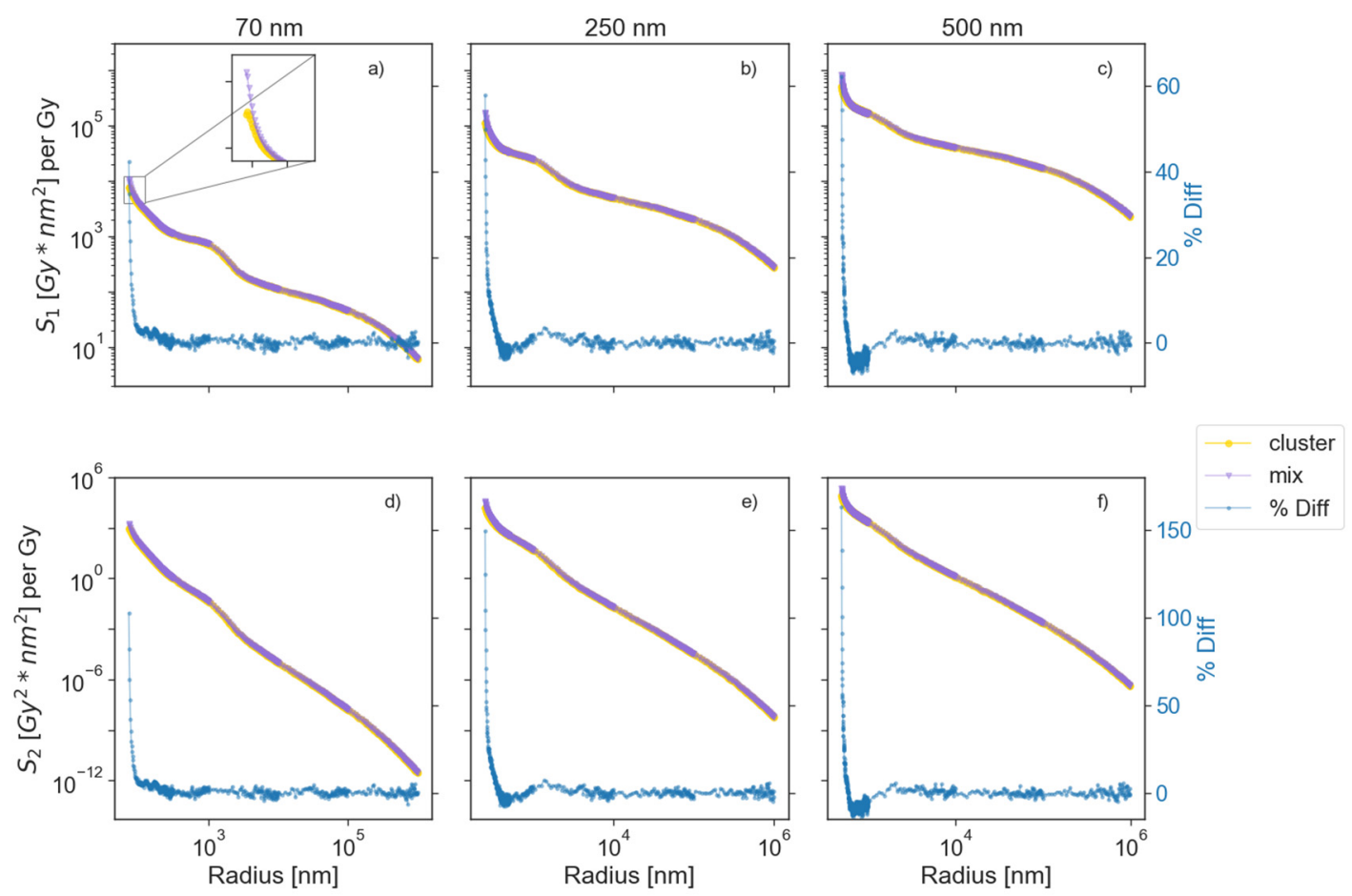

Figure 3. Upper row (a-c) shows the behaviors of $S_{1}$ for a cluster of GNPs and a MixNP for all sizes, along with the \%Diff between them (blue). $S_{1}$ represents the dose in a spherical shell and thickness of $d r$ integrated over the surface of a circle of radius $r$. Due to the slow decrease of $S_{1}$ along the radius, we scored the dose far away from the nanoparticle. Lower row $(\mathbf{d}-\mathbf{f})$ shows the change in $S_{2}$ along the radial distances. $S_{2}$ is defined similarly to $S_{1}$, but using the dose squared in the surface integral. Due to the closeness of the values of $S_{1}$ for the full cluster and a MixNP, a zoomed-in view was added in (a).

In this work, the cell was approximated as two concentric spherical regions (nucleus and cytoplasm). Our model allowed for a study of the change in the average number of lethal lesions when the cluster/MixNP was placed at different positions inside the cell. Furthermore, our model also makes it feasible to consider both regions as our sensitive volume. Nevertheless, previous studies where GNPs were injected into several cell lines [29,30], associate a low probability to nanoparticle entry into the nucleus, therefore, this study only 
comprised of placements of the nanoparticle clusters outside the nucleus. More recent imaging studies [31] have shown that monodispersed nanoparticles can in fact penetrate the nucleus. In this case, however, it is clear that the approximation used here will break down since it considers the cluster of nanoparticles to be biologically inert. For that case, a more complex formalism has been already provided [28], which can be used to predict dose enhancements, as has already been illustrated [31]. In addition, clusters/MixNPs were also considered to be far outside the cell in order to study the influence of nanoparticles placed inside the surrounding cells on the lethal lesions created in the $V_{\text {sens }}$.

When $V_{\text {sens }}$ was assigned to the cytoplasm, the parameters of interest in the biological stage were $I_{1} c y t$ and $I_{2} c y t$, but those changed to $I_{1} n u c$ and $I_{2} n u c$ when the nucleus was considered as $V_{\text {sens }}$. Figure 4 shows the changes of $I_{1}$ and $I_{2}$ when the cluster/MixNP was placed at different locations inside the cell. Figure $4 \mathrm{~b}-\mathrm{d}, \mathrm{h}-\mathrm{j}$ consider the cytoplasm as $V_{\text {sens }}$, while Figure $4 \mathrm{e}-\mathrm{g}, \mathrm{k}-\mathrm{m}$ consider the nucleus as the $V_{\text {sens }}$. Figure 4 also shows the \%Diff between the parameters of interest for all scenarios.
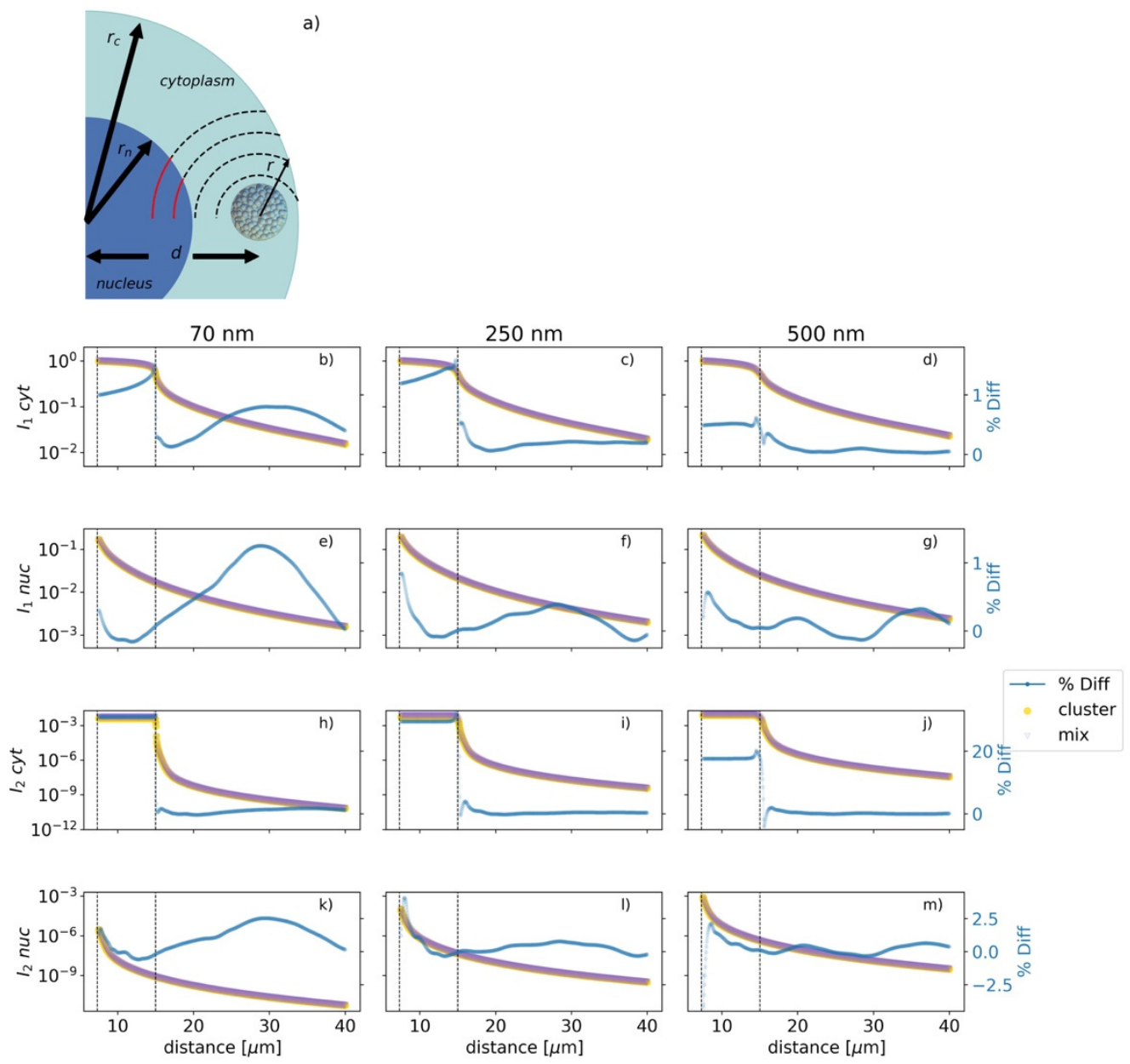

Figure 4. (a) The cell used in this work was modelled as an outer and an inner sphere representing the cytoplasm and nucleus, with radii of $r_{c}=15$ and $r_{n}=7 \mu \mathrm{m}$, respectively. The cluster/MixNP was placed at different positions $d$ from the center of the cell for the evaluation of $I_{1}$ and $I_{2}$ for all cluster sizes. (b-g) show $I_{1}$ for a MixNP and a full cluster along with the \%Diff between them when considering the cytoplasm and the nucleus as $V_{\text {sens }}$, respectively. (h-m) describe $I_{2}$ for a MixNP and a full cluster as well as the \%Diff between them when considering the cytoplasm and the nucleus as $V_{\text {sens }}$, respectively. All the curves in the same column are normalized with respect to the $I_{1} c y t$ for the cluster of GNPs. The two black dotted lines correspond to the distances of 7.5 and $15 \mu \mathrm{m}$.

As shown in Figure $4 \mathrm{~b}-\mathrm{d}$, we observed that the \%Diff for the $I_{1}$ cyt rose between the distances of 7 and $15 \mu \mathrm{m}$ (the nucleus and cytoplasm radii, respectively), and reached a 
peak at $15 \mu \mathrm{m}$, with values of 1.52, 1.53, and 0.62 for the cluster sizes of 70, 250, and $500 \mathrm{~nm}$, respectively. This peak corresponded to the cluster spanning the cell membrane and hence does not represent a physically realistic scenario. Figure $4 \mathrm{~h}-\mathrm{j}$ shows that the $\%$ Diff for the $I_{2}$ cyt remained steady inside the cell and that its peak values at the cell edge were 32.7, 32.8 , and 20.2 for the cluster sizes of 70, 250, and $500 \mathrm{~nm}$, respectively, dropping sharply after that. $I_{1} c y t$ had a steady decline inside the cell volume for the MixNP and the full cluster and declined at a faster rate beyond the cell boundary. $I_{2} c y t$ remained steady inside the cell and plummeted at $15 \mu \mathrm{m}$, decreasing for further distances in both the MixNP and the full cluster scenarios.

Figure $4 \mathrm{e}-\mathrm{g}, \mathrm{k}-\mathrm{m}$ illustrate the parameters of interest when the nucleus was considered as the sensitive volume. The position of the maximum values for the \% Diff for the $I_{1}$ nuc varied between cluster sizes, being localized at $28.85,7.55$, and $8.10 \mu \mathrm{m}$ for the 70,250 , and $500 \mathrm{~nm}$ cluster sizes, respectively; while the maximum values for the \%Diff for the $I_{2} n u c$ were $29.4,0.8$, and $8.55 \mu \mathrm{m}$ for the 70,250 , and $500 \mathrm{~nm}$ sizes, respectively. $I_{1} n u c$ and $I_{2} n u c$ decreased steadily for all distances, having their maximum at the nucleus surface.

\section{Discussion}

In this work, we investigated the differences in the cell damage generated by the activation of a cluster of gold nanoparticles irradiated by a 6 MV TrueBeam Varian Linac compared to a homogeneous mixture of gold and water irradiated under the same conditions. The data from Figure 4 illustrate that for both scenarios, the cluster of GNPs and the MixNP, the main parameter driving the biological stage for all cluster sizes was $I_{1}$, which is several orders of magnitude higher than $I_{2}$. This was true for both of the sensitive volumes considered in this work: the cytoplasm and the nucleus. Furthermore, $I_{1} c y t$ was one order of magnitude higher than $I_{1} n u c$, implying that the majority of the energy deposition occurred outside the nucleus. Thus, by neglecting $I_{2}$ from Equation (3) and rearranging the terms, we can define the radio sensitizer enhancement factor $(S E F)$ as:

$$
S E F=I_{1} \frac{\alpha D+2 \beta D^{2}}{V_{\text {sens }}\left(\alpha D+\beta D^{2}\right)}
$$

where $\alpha$ and $\beta$ are constant parameters depending on the cell line, while $V_{\text {sens }}$ depends on the sensitive volume of the cell in which we are counting the number of lethal lesions, leaving $I_{1}$ as the main parameter driving the change to the surviving fraction generated by the nanoparticle.

The high value of the \%Diff at the physical stage shown in Figure 2 translates into the biological stage as high values \%Diff values for $I_{2} n u c$ and $I_{2} c y t$, as illustrated in Figure 4 . However, $I_{1}$ cyt drives the biological stage, as mentioned above. The $\%$ Diff fell to $\sim 1.5 \%$ for the 70 and $250 \mathrm{~nm}$ clusters and fell to less than $1 \%$ for the $500 \mathrm{~nm}$ cluster. Therefore, the number of lethal lesions in the cell created by the activation of a full cluster was satisfactorily approximated by the MixNP. This novel result implies two things: firstly, it provides a ready reckoning of how uptake in a microscope image (without resolving individual nanoparticles) can be related to enhancement, and secondly, it facilitates the reduction of the many-body problem to one body when considering the interior of the cluster as not biologically active, considerably reducing the complexity of the problem and saving computational resources. This study estimates a $50 \%$ reduction of computational time for the Monte Carlo simulations at nanoscale when a considering a MixNP instead of a full cluster of GNPs for calculations of PHSP2.

Although our simulations were confined to spherically symmetric clusters, this was simply for computational convenience, as it provided simple closed forms for the relationships expressed in Equations (4)-(7). Our main conclusion is that using the homogenous MixNP results in the same predicted biological effects as the cluster of individual NPs, provided the effect is being considered outside of the cluster. It is immediately obvious that this result extends to any shape of cluster since it can be well represented by an ensemble 
of spherical clusters, i.e., there is a super-position principle one can apply to the evaluation of $I_{1}$ and $I_{2}$, approximating any shape of cluster by means of many spherical ones.

The increase of the \%Diff between a cluster and a MixNP near the nanoparticle surface in the physical stage, as shown in Figure 2, was associated with the simplified modelling for the MixNP as a perfectly homogeneous mix, causing an excess of electrons at the surface. By contrast, with a real cluster the average density decreases slightly at the surface due to the amount of interstitial water between the nanoparticles. The high values of the DEF created by the MixNP agree with previous work by Zhan and Koger [32,33], who obtained $\sim 30 \%$ for both studies using mono-energetic gamma beams. Nevertheless, by investigating this phenomenon in more detail, we observed that more complex models for the MixNP could decrease the \%Diff considerably at the physical stage, as is shown in the Supplementary Materials. This quantity decreased by approximately one order of magnitude by considering a water shell surrounding the MixNP. However, the use of more complex models was not necessary for this work because the \%Diff for the biological $I_{1}$ became negligible when the LEM was applied and integrated over the sensitive volume.

Due to the current lack of knowledge on the radiolysis when GNPs are present during irradiation, the chemistry stage was out of scope in this work. However, we cannot discard the possibility of a higher value of the \%Diff for $I_{1}$ and $I_{2}$ in the biological stage when the production of radicals is taken into account.

Previous experiments support the evidence that the nanoparticles tend to cluster inside the cell [9-11], suggesting this study represents a more realistic scenario than those interpreting GNPs with a uniform random distribution inside the cell. Furthermore, the approach used to model the aggregation of nanoparticles using a spherical geometry is supported by previous work in the literature [34] where the sizes of the clusters were measured by their diameters using TEM scan.

The maximum value for the DEF observed in this work at the physical stage $(\sim 20)$ when the GNPs of all cluster sizes were activated with $6 \mathrm{MV}$ Linac irradiation is in agreement with previous studies [35,36], where the maximum values of the DEF were found to be between 8 and 17. The dependency observed on the relationship between the position of the cluster inside the cell and the enhancement of DNA double-strand break (DBS) formation inside the nucleus during irradiation was also observed in previous work based on computational simulations [28,37]. In these studies, the cells were irradiated under similar conditions to work. Furthermore, the results of [38-40], where various cell lines were investigated using different techniques such as radiation-induced foci of $\gamma$-H2AX staining and immunohistochemistry, confirm the trend for $I_{1} n u c$, as shown in Figure 4, to damage cell nuclei at mega-voltage energies.

Clearly, for the model presented here to be valid, the size of each nanoparticle needs to be significantly smaller than the overall size of the cluster or, equivalently, there needs to be many nanoparticles in the cluster. Provided this condition is met, there is an interesting and perhaps slightly counterintuitive conclusion-the biological effect is only very weakly dependent on the nanoparticle size (see Figure 4). It should, however, be noted that we factored the dominant nanoparticle size dependence out of our simulations, since we constructed clusters containing a constant number of nanoparticles and a constant total fractional density of gold, i.e., bigger nanoparticles were used to constitute bigger clusters.

Although the model presented is a macroscopic one, it pertains at the single-cell level, as illustrated in Figure 4. Hence, it is applicable at either the cellular level or at the patient/organ level when applied to radiotherapy. However, in the former case, the validity of the model rests on the assumption that the biologically active region is entirely outside the cluster. This assumption is not always valid, e.g., see [31].

The similarities between the $I_{1}$ curves for both the MixNP and the full cluster for cases where the critical target was either in the nucleus $\left(I_{1} n u c\right.$ of Figure 4$)$ or the cytoplasm $\left(I_{1}\right.$ cyt of Figure 4) show that that there was an equivalence of the biological effect induced by irradiation of clusters of heavy-atom nanoparticles and homogeneous heavy-atom-water mixtures for either type of target. Furthermore, one could take a linear combination of 
$I_{1} n u c$ and $I_{1} c y t$ corresponding to the different weighting factors for the nuclear and cytoplasmic targets and still have nearly equivalent results for the MixNP and full cluster cases. Hence, the conclusion regarding the equivalence of the biological effect in these two scenarios is robust, even though there is ongoing controversy regarding the nature of the critical targets, as was highlighted in the introduction [12-15].

\section{Conclusions}

We implemented a multiscale approach by applying the LEM to fully simulated clusters of GNPs using the Monte Carlo technique and irradiated them in a clinically relevant scenario. The resultant biological effect in cells is well represented through a single parameter, $I_{1}$, which describes the increase in the number of lethal lesions for the two sensitive volumes studied in this work. The predicted cell damage generated by such clusters is essentially the same as for a homogeneous mixture of gold and water. This equivalence is useful both to intuiting the effect of measured nanoparticle distributions and also to performing a rapid computational evaluation of these effects.

Supplementary Materials: More information on the modelling of the complex MiXNP mentioned in Results section is available online at https:/ /www.mdpi.com/article/10.3390/cancers13092034/s1, Figure S1: Visualization of the heterogenous MixNP, Figure S2: DEF and \%Diff (blue) between the cluster and the heterogenous MixNP for all sizes, Figure S3: Box plot comparing the \%Diff between the cluster and the heterogeneous/homogeneous MixNP for all sizes, Table S1: Set of optimized parameters used to model the heterogeneous MixNP for all sizes.

Author Contributions: Conceptualization, F.J.C. and B.V.-B.; methodology, F.J.C., J.R.-M. and B.V.-B.; software, F.J.C. and B.V.-B.; validation, F.J.C. and B.V.-B., J.R.-M.; formal analysis, F.J.C. and B.V.-B.; investigation, F.J.C., J.R.-M. and B.V.-B.; resources, F.J.C., J.R.-M. and B.V.-B.; data curation, B.V.-B.; writing — original draft preparation, B.V.-B.; writing—review and editing, F.J.C., J.R.-M. and B.V.-B.; visualization, F.J.C. and B.V.-B.; supervision, F.J.C.; project administration, B.V.-B.; funding acquisition, F.J.C. All authors have read and agreed to the published version of the manuscript.

Funding: J.R.-M was funded by National Institutes of Health/National Cancer Institute (NIH/NCI) with grant no. R01 CA187003: "TOPAS-NBIO: A Monte Carlo tool for radiation biology research". B.V.B and F.J.C were funded by the Engineering and Physical Science Research Council (EPSRC) with the grant No. EP/R030677/1: "Clinical Adaptative Radiation Transport Algorithms".

Data Availability Statement: Computer codes used to perform these calculations will be made publicly available through the University of Manchester's institutional repository upon its acceptance for publication.

Acknowledgments: The authors would like to acknowledge the assistance given by the Research IT group and the use of the Computational Shared Facility at University of Manchester. We acknowledge the support of The University of Manchester's Dalton Cumbrian Facility (DCF), a partner in the National Nuclear User Facility, the EPSRC UK National Ion Beam Centre and the Henry Royce Institute.

Conflicts of Interest: The authors declare no conflict of interest.

\section{References}

1. Rippel, R.A.; Seifalian, A.M. Gold Revolution -Gold nanoparticles for modern medicine and surgery. J. Nanosci. Nanotechnol. 2011, 11, 7340-7348. [CrossRef]

2. Ghosh, P.; Hang, G.; De, M.; Chae, K.K.; Rotello, V.M. Gold nanoparticles in delivery applications. Adv. Drug Deliv. Rev. 2008, 60, 1307-1315. [CrossRef]

3. Hainfeld, J.F.; Dilmanian, F.A.; Zhong, Z.; Slatkin, D.N.; Kalef-Ezra, J.A.; Smilowitz, H.M. Gold nanoparticles enhance the radiation therapy of a murine squamous cell carcinoma. Phys. Med. Biol. 2010, 55, 3045-3059. [CrossRef]

4. Siddique, S.; Chow, J.C.L. Application of Nanomaterials in Biomedical Imaging and Cancer Therapy. Nanomaterials 2020, 10, 1700. [CrossRef] [PubMed]

5. Moore, J.A.; Chow, J.C.L. Recent progress and applications of gold nanotechnology in medical biophysics using artificial intelligence and mathematical modelling. Nano Express 2021, 2, 022001. [CrossRef] 
6. McMahon, S.J.; Hyland, W.B.; Muir, M.F.; Coulter, J.A.; Jain, S.; Butterworth, K.T.; Schettino, G.; Dickson, G.R.; Hounsell, A.R.; O'Sullivan, J.M.; et al. Nanodosimetric effects of gold nanoparticles in megavoltage radiation therapy. Radiother. Oncol. 2011, 100, 412-416. [CrossRef] [PubMed]

7. Lechtman, E.; Chattopadhyay, N.; Cai, Z.; Mashouf, S.; Reilly, R.; Pignol, J.P. Implications on clinical scenario of gold nanoparticle radiosensitization in regards to photon energy, nanoparticle size, concentration and location. Phys. Med. Biol. 2011, 56, $4631-4647$. [CrossRef] [PubMed]

8. Butterworth, K.T.; McMahon, S.J.; Currell, F.J.; Prise, K.M. Physical basis and biological mechanisms of gold nanoparticle radio sensitization. Nanoscale 2012, 4, 4830-4838. [CrossRef]

9. Marill, J.; Anesary, N.M.; Zhang, P.; Vivet, S.; Borgh, E.; Levy, L.; Pottier, A. Hafnium oxide nanoparticles: Toward an in vitro predictive biological effect? Radiat. Oncol. 2014, 9, 150. [CrossRef]

10. Chithrani, B.D.; Ghazani, A.A.; Chan, W.C.W. Determining the Size and Shape Dependence of Gold Nanoparticle Uptake into Mammalian Cells. Nano Lett. 2006, 6, 662-668. [CrossRef]

11. Arnida, M.A.; Ghandehari, H. Cellular uptake and toxicity of gold nanoparticles in prostate cancer cells: A comparative study of rods and spheres. J. Appl. Toxicol. 2009, 30, 212-217. [CrossRef]

12. Pagáčová, E.; Štefančíková, L.; Schmidt-Kaler, F.; Hildenbrand, G.; Vičar, T.; Depeš, D.; Lee, J.; Bestvater, F.; Lacombe, S.; Porcel, E.; et al. Challenges and Contradictions of Metal Nano-Particle Applications for Radio-Sensitivity Enhancement in Cancer Therapy. Int. J. Mol. Sci. 2019, 20, 588. [CrossRef]

13. Hausmann, M.; Falk, M.; Neitzel, C.; Hofmann, A.; Biswas, A.; Gier, T.; Falkova, I.; Heermann, D.W.; Hildenbrand, G. Elucidation of the Clustered Nano-Architecture of Radiation-Induced DNA Damage Sites and Surrounding Chromatin in Cancer Cells: A Single Molecule Localization Microscopy Approach. Int. J. Mol. Sci. 2021, 22, 3636. [CrossRef]

14. Taggart, L.E.; McMahon, S.J.; Currell, F.J.; Prise, K.M.; Butterworth, K.T. The role of mitochondrial function in gold nanoparticle mediated radiosensitisation. Cancer Nanotechnol. 2014, 5, 1-12. [CrossRef]

15. Štefančíková, L.; Lacombe, S.; Salado, D.; Porcel, E.; Pagáčová, E.; Tillement, O.; Lux, F.; Depeš, D.; Kozubek, S.; Falk, M. Effect of gadolinium-based nanoparticles on nuclear DNA damage and repair in glioblastoma tumor cells. J. Nanobiotechnol. 2016, 14, 1-15. [CrossRef] [PubMed]

16. Garnica-Garza, H.M. Contrast-enhanced radiotherapy: Feasibility and characteristics of the physical absorbed dose distribution for deep-seated tumors. Phys. Med. Biol. 2009, 54, 5411-5425. [CrossRef] [PubMed]

17. Cho, S.H. Estimation of tumour dose enhancement due to gold nanoparticles during typical radiation treatments: A preliminary Monte Carlo study. Phys. Med. Biol. 2005, 50, 163-173. [CrossRef] [PubMed]

18. Chow, J.C.L. Recent progress in Monte Carlo simulation on gold nanoparticle radiosensitization. AIMS Biophys. 2018, 5, 231-244. [CrossRef]

19. Constantin, M.; Constantin, D.; Keall, P.J.; Narula, A.; Svatos, M.; Perl, J. Linking computer-aided design (CAD) to Geant4-based Monte Carlo simulations for precise implementation of complex treatment head geometries. Phys. Med. Biol. 2010, 55, 211-220. [CrossRef]

20. Lin, Y.; McMahon, S.J.; Scarpelli, M.; Paganetti, H.; Schuemann, J. Comparing gold nano-particle enhanced radiotherapy with protons, megavoltage photons and kilovoltage photons: A Monte Carlo simulation. Phys. Med. Biol. 2014, 59, 7675-7689. [CrossRef] [PubMed]

21. Bernal, M.A.; Bordage, M.C.; Brown, J.M.C.; Davídková, M.; Delage, E.; El Bitar, Z.; Enger, S.; Francis, Z.; Guatelli, S.; Ivanchenko, V.N.; et al. Track structure modelling in liquid water: A review of the Geant4-DNA very low energy extension of the Geant4 Monte Carlo simulation toolkit. Phys. Med. 2015, 31, 861-874. [CrossRef]

22. Incerti, S.; Ivanchenko, A.; Karamitros, M.; Mantero, A.; Moretto, P.; Tran, H.N.; Mascialino, B.; Champion, C.; Ivanchenko, V.N.; Bernal, M.A.; et al. Comparison of Geant4 very low energy cross section models with experimental data in water. Med. Phys. 2010, 37, 4692-4708. [CrossRef]

23. Incerti, S.; Douglass, M.; Penfold, S.; Guatelli, S.; Bezak, E. Review of Geant4-DNA applications for micro and nanoscale simulations. Phys. Med. 2016, 32, 1187-11200. [CrossRef] [PubMed]

24. Perl, J.; Shin, J.; Schümann, J.; Faddegon, B.; Paganetti, H. TOPAS: An innovative proton Monte Carlo platform for research and clinical applications. Med. Phys. 2012, 39, 6818-6837. [CrossRef]

25. Agostinelli, S.; Allison, J.; Amako, K.; Apostolakis, J.; Araujo, H.; Arce, P.; Asai, M.; Axen, D.; Banerjee, S.; Barrand, G.; et al. Geant4-A simulation toolkit. Nucl. Instrum. Methods Phys. Res. A 2003, 506, 250-303. [CrossRef]

26. Currell, F.J.; Villagomez-Bernabe, B. Physical and Chemical Processes for Gold Nanoparticles and Ionizing Radiation in Medical Contexts. In Gold Nanoparticles for Physics, Chemistry and Biology, 2nd ed.; Louis, C., Pluchery, O., Eds.; World Scientific: London, UK, 2017; pp. 509-536, ISBN 1786341247.

27. Scholz, M.; Kraft, G. Track structure and the calculation of biological effects of heavy charged particles. Adv. Space Res. 1996, 8, 5-14. [CrossRef]

28. Villagomez-Bernabe, B.; Currell, F.J. Physical radiation enhancement effects around clinically relevant clusters of nano agents in biological systems. Sci. Rep. 2019, 9, 8156. [CrossRef] [PubMed]

29. Coulter, J.A.; Jain, S.; Butterworth, K.T.; Taggart, L.E.; Dickson, G.R.; McMahon, S.J.; Hyland, W.B.; Muir, M.F.; Trainor, C.; Hounsell, A.R.; et al. Cell type-dependent uptake, localization, and cytotoxicity of $1.9 \mathrm{~nm}$ gold nanoparticles. Int. J. Nanomed. 2012, 7, 2673-2685. [CrossRef] [PubMed] 
30. Botchway, S.W.; Coulter, J.A.; Currell, F.J. Imaging intracellular and systemic in vivo gold nanoparticles to enhance radiotherapy. Br. J. Radiol. 2015, 88, 2-13. [CrossRef] [PubMed]

31. McCulloch, A.; Bennie, L.; Coulter, J.A.; McCarthy, H.O.; Dromey, B.; Grimes, D.R.; Quinn, P.; Villagomez-Bernabe, B.; Currell, F. Nuclear uptake of gold nanoparticles deduced using dual-angle X-Ray fluorescence mapping. Part. Part. Syst. Charact. 2019, 36, 1-9. [CrossRef]

32. Zhang, S.X.; Gao, J.; Buchholz, T.A.; Wang, Z.; Salehpour, M.R.; Drezek, R.A.; Yu, T.K. Quantifying tumor-selective radiation dose enhancements using gold nanoparticles: A Monte Carlo simulation study. Biomed. Microdevices 2009, 11, 925-933. [CrossRef] [PubMed]

33. Koger, B.; Kirkby, C. A method for converting dose-to-medium to dose-to-tissue in Monte Carlo studies of gold nanoparticleenhanced radiotherapy. Phys. Med. Biol. 2016, 61, 2014-2024. [CrossRef] [PubMed]

34. Kim, J.; Chun, S.H.; Amornkitbamrung, L.; Song, C.; Yuk, J.S.; Ahn, S.Y.; Kim, B.W.; Lim, Y.T.; Oh, B.-K.; Um, S.H. Gold nanoparticle clusters for the investigation of therapeutic efficiency against prostate cancer under near-infrared irradiation. Nano Convergence 2020, 7, 1-9. [CrossRef] [PubMed]

35. Jones, B.L.; Krishnan, S.; Cho, S.H. Estimation of microscopic dose enhancement factor around gold nanoparticles by Monte Carlo calculations. Med. Phys. 2010, 37, 3809-3816. [CrossRef] [PubMed]

36. Tsiamas, P. Impact of beam quality on megavoltage radiotherapy treatment techniques utilizing gold nanoparticles for dose enhancement. Phys. Med. Biol. 2013, 58, 451-464. [CrossRef] [PubMed]

37. Liu, R.; Zhao, T.; Zhao, X.; Reynoso, F.J. Modeling gold nanoparticle radiosensitization using a clustering algorithm toquantitate DNA double-strand breaks with mixed-physics Monte Carlo Simulation. Med. Phys. 2019, 46, 5314-5325. [CrossRef]

38. Liu, C.J. Enhancement of cell radiation sensitivity by pegylated gold nanoparticles. Phys. Med. Biol. 2010, 55, 931-945. [CrossRef]

39. Berbeco, R.I.; Korideck, H.; Ngwa, W.; Kumar, R.; Patel, J.; Sridhar, S.; Johnson, S.; Price, B.D.; Kimmelman, A.; Makrigiorgos, G.M. DNA damage enhancement from gold nanoparticles for clinical MV photon beams. Radiat. Res. 2012, 178, 604-608. [CrossRef]

40. Chithrani, D.B.; Jelveh, S.; Jalali, F.; Van Prooijen, M.; Allen, C.; Bristow, R.G.; Hill, R.P.; Jaffray, D.A. Gold Nanoparticles as Radiation Sensitizers in Cancer Therapy. Radiat. Res. 2010, 173, 719-728. [CrossRef] 